\title{
Acyclic coefficient systems on buildings
}

\author{
Elmar Grosse-Klönne \\ In memory of Prof. Hans Joachim Nastold
}

\begin{abstract}
For cohomological (respectively homological) coefficient systems $\mathcal{F}$ (respectively $\mathcal{V}$ ) on affine buildings $X$ with Coxeter data of type $\widetilde{A}_{d}$, we give for any $k \geqslant 1$ a sufficient local criterion which implies $H^{k}(X, \mathcal{F})=0$ (respectively $H_{k}(X, \mathcal{V})=0$ ). Using this criterion we prove a conjecture of de Shalit on the acyclicity of coefficient systems attached to hyperplane arrangements on the Bruhat-Tits building of the general linear group over a local field. We also generalize an acyclicity theorem of Schneider and Stuhler on coefficient systems attached to representations.
\end{abstract}

\section{Introduction}

Let $X$ be an affine building whose apartments are Coxeter complexes attached to Coxeter systems of type $\widetilde{A}_{d}$ and let $\mathcal{F}$ be a cohomological coefficient system (CCS) on $X$. The purpose of this paper is to give a local criterion which assures that for a given $k \geqslant 1$ the cohomology group $H^{k}(X, \mathcal{F})$ vanishes (similarly for homological coefficient systems) (HCSs).

For a sheaf $\mathcal{G}$ on a topological space $Y$ it is well known that $H^{k}(Y, \mathcal{G})=0$ for all $k \geqslant 1$ if $\mathcal{G}$ is flasque, i.e. if all restriction maps $\mathcal{G}(U) \rightarrow \mathcal{G}(V)$ for open $V \subset U \subset Y$ are surjective. We are looking for an adequate notion of 'flasque' CCS on $X$.

If $d=1$ the same condition works: if the restriction map $\mathcal{F}(\sigma) \rightarrow \mathcal{F}(\tau)$ for any 0 -simplex $\sigma$ contained in the 1 -simplex $\tau$ is surjective, then $H^{1}(X, \mathcal{F})=0$. This is easily seen using the contractibility of $X$. However, if $d>1$ the surjectivity of $\mathcal{F}(\sigma) \rightarrow \mathcal{F}(\tau)$ for any $(k-1)$-simplex $\sigma$ contained in the $k$-simplex $\tau$ does not guarantee $H^{k}(X, \mathcal{F})=0$. The other naive transposition of the flasqueness concept from topological spaces to buildings would be to require for any $(k-1)$-simplex $\sigma$ the surjectivity of $\mathcal{F}(\sigma) \rightarrow \prod_{\tau} \mathcal{F}(\tau)$, taking the product over all $k$-simplices $\tau$ containing $\sigma$. This would indeed force $H^{k}(X, \mathcal{F})=0$, but would also be a completely useless criterion: for example, it would not be satisfied by a constant $\operatorname{CCS} \mathcal{F}$ (of which we know $H^{k}(X, \mathcal{F})=0$, by the contractibility of $X$ ).

Let us describe our criterion $\mathcal{S}(k)$. We fix an orientation of $X$. It defines a cyclic ordering on the set of vertices of any simplex, hence a true ordering on the set of vertices of any pointed simplex. To a pointed $(k-1)$-simplex $\widehat{\eta}$ we associate the set $N_{\widehat{\eta}}$ of all vertices $z$ for which $(z, \widehat{\eta})$ (i.e. $z$ as the first vertex) is an ordered $k$-simplex (in the previously qualified sense). We define what it means for a subset $M_{0}$ of $N_{\widehat{\eta}}$ to be stable with respect to $\widehat{\eta}$ (if, for example, $d=1$ the condition is $\left|M_{0}\right| \leqslant 1$ ). Our criterion $\mathcal{S}(k)$ which assures $H^{k}(X, \mathcal{F})=0$ is then that for any such $\widehat{\eta}$ and for any subset $M_{0}$

Received 14 February 2004, accepted in final form 26 May 2004, published online 21 April 2005.

2000 Mathematics Subject Classification 20E42.

Keywords: building, Bruhat-Tits building, coefficient system, cohomology, hyperplane arrangement, Orlik-Solomon algebra.

This journal is (C) Foundation Compositio Mathematica 2005. 


\section{E. Grosse-KLÖNNE}

of $N_{\widehat{\eta}}$, stable with respect to $\widehat{\eta}$, the sequence

$$
\mathcal{F}(\eta) \longrightarrow \prod_{z \in M_{0}} \mathcal{F}(\{z\} \cup \eta) \longrightarrow \prod_{z, z^{\prime} \in M_{0}} \mathcal{F}\left(\left\{z, z^{\prime}\right\} \cup \eta\right)
$$

is exact (where in the target of the second arrow the product is over pairs $z, z^{\prime} \in M_{0}$ of incident vertices). For example, a constant CCS $\mathcal{F}$ satisfies $\mathcal{S}(k)$.

Having fixed a vertex $z_{0}$, the central ingredient in the proof is then a certain function $i$ on the set $X^{0}$ of vertices which measures the combinatorical (not Euclidean) distance from $z_{0}$; it depends on our chosen orientation.

Dual to $\mathcal{S}(k)$ we describe a criterion $\mathcal{S}^{*}(k)$ which guarantees $H_{k}(X, \mathcal{V})=0$ for HCSs $\mathcal{V}$ on $X$.

The basic example is, of course, the case where $X$ is the Bruhat-Tits building of $\mathrm{PGL}_{d+1}(K)$ for a local field $K$. Our original motivation for developing the criterion $\mathcal{S}(k)$ was the following. In [Sch92], Schneider defined a certain class of $\mathrm{SL}_{d+1}(K)$-representations, the 'holomorphic discrete series representations', as the global sections of certain equivariant vector bundles on Drinfel'd's symmetric space $\Omega_{K}^{(d+1)}$ of dimension $d$ over $K$. In a subsequent paper [Gro04], for any such vector bundle $V$, we will construct an integral model $\mathcal{V}$ as an equivariant coherent sheaf on the formal $\mathcal{O}_{K}$-scheme $\Omega_{\mathcal{O}_{K}}^{(d+1)}$ underlying $\Omega_{K}^{(d+1)}$. Using the criterion $\mathcal{S}(k)$ and the close relation between $\Omega_{K}^{(d+1)}$ and $X$, we will show that if $V$ is strongly dominant (in a suitable sense), then $H^{k}\left(\Omega_{\mathcal{O}_{K}}^{(d+1)}, \mathcal{V}\right)=0$ for all $k \geqslant 1$. Examples for such $\mathcal{V}$ are the terms of the logarithmic de Rham complex of $\Omega_{\mathcal{O}_{K}}^{(d+1)}$.

Here we present two other applications. The first is a proof of a conjecture of de Shalit on $p$-adic CCSs of Orlik-Solomon algebras. For an arbitrary field $K$, the assignment of the OrlikSolomon algebra $A$ to a hyperplane arrangement $W$ in $\left(K^{d+1}\right)^{*}$, the complement of a finite set $\mathcal{A} \subset \mathbb{P}\left(K^{d+1}\right)$ of hyperplanes in $\left(K^{d+1}\right)^{*}$, is a classical theme. $A$ is defined combinatorically in terms of the hyperplanes and turns out to be isomorphic with the cohomology ring of $W$. Of course $K$ may also be a local field. However, de Shalit [deS01] discovered that one can go further and give the story a genuinely $p$-adic flavour. Namely, if $K$ is a local field he allowed $\mathcal{A} \subset \mathbb{P}\left(K^{d+1}\right)$ to be infinite. He did not assign a single Orlik-Solomon algebra to $\mathcal{A}$ but a CCS system $A=A(\cdot)$ of Orlik-Solomon algebras on the Bruhat-Tits building $X$ of $\operatorname{PGL}_{d+1}(K)$ which then should play the role of a cohomology ring of the 'hyperplane arrangement' defined by $\mathcal{A}$. The algebra $A(\sigma)$ for a $j$-simplex $\sigma$ is closely related to a suitable tensor product of Orlik-Solomon algebras for finite hyperplane arrangements in $k$-vector spaces, for $k$ the residue field of $K$. de Shalit conjectures that these beautiful CCSs are acyclic in positive degrees, $H^{k}(X, A)=0$ for all $k \geqslant 1$. He proved the conjecture for any $\mathcal{A}$ if $d \leqslant 2$. For arbitrary $d$ he proved it if $\mathcal{A}$ is the full set $\mathbb{P}\left(K^{d+1}\right)$ of all $K$-rational hyperplanes, and Alon [Alo] proved it if $\mathcal{A}$ is finite. Here we give a proof for all $\mathcal{A}$ and $d$ by showing that $A$ always satisfies $\mathcal{S}(k)$.

In fact we prove a version with arbitrary coefficient ring: while in [deS01] the coefficient ring of the CCS $A$ is $K$, we allow an arbitrary coefficient ring $R$, e.g. also $R=\mathbb{Z}$ or $R=k$. While de Shalit's proof in the case $d \leqslant 2$ also works for arbitrary $R$, his proof in the case $\mathcal{A}=\mathbb{P}\left(K^{d+1}\right)$ but $d$ arbitrary, which is by reduction to the main result of [SS93], does not work for coefficient rings $R$ other than characteristic zero fields. We explain why this improvement for $\mathcal{A}=\mathbb{P}\left(K^{d+1}\right)$ and $R=\mathbb{Z}$ should have an application to a problem on $p$-adic Abel-Jacobi mappings raised in [RX03].

The second application we describe is concerned with the technique of Schneider and Stuhler to spread out representations of $\mathrm{GL}_{d+1}(K)$ as HCSs on the Bruhat-Tits building of $\mathrm{PGL}_{d+1}(K)$. For a $\mathrm{GL}_{d+1}(K)$-representation on a (not necessarily free) $\mathbb{Z}\left[\frac{1}{p}\right]$-module $V$ (where $p=\operatorname{char}(k)$ ) which is generated by its vectors fixed under a principal congruence subgroup $U^{(n)}$ of some level $n>1$, we prove that the chain complex of the corresponding HCS is a resolution of $V$. For fields of characteristic zero as coefficient ring (instead of $\mathbb{Z}\left[\frac{1}{p}\right]$ ) this is the main result of [SS93], where, 


\section{ACYCLIC COEFFICIENT SYSTEMS ON BUILDINGS}

however, $n=1$ is allowed. While the proof in [SS93] uses the Bernstein-Borel-Matsumoto theory, we do not need any representation theoretic input whatsoever.

\section{The criterion}

Let $d \geqslant 1$ and let $X$ be an affine building whose apartments are Coxeter complexes attached to Coxeter systems of type $\widetilde{A}_{d}$. We refer to [Bro89] for the basic definitions and properties of buildings. For $0 \leqslant j \leqslant d$ we denote by $X^{j}$ the set of $j$-simplices. We generally identify a $j$-simplex with its set of vertices.

We fix an orientation of $X$. It distinguishes for any simplex a cyclic ordering on its set of vertices. A pointed $k$-simplex is an enumeration of the set of vertices of a $k$-simplex in its distinguished cyclic ordering; we write it as an ordered $(k+1)$-tuple of vertices.

For an apartment $A$ in $X$ we will slightly abuse notation by not distinguishing between $A$ and its geometric realization $|A|$. There is (see [Bro89, p. 148]) an isomorphism of $A$ with $\mathbb{R}^{d+1} / \mathbb{R} \cdot(1, \ldots, 1$ ), we view it here as an identification, such that, if $\left\{e_{0}, \ldots, e_{d}\right\}$ denotes the standard basis of $\mathbb{R}^{d+1}$ the following holds:

(a) the set of vertices in $A$ is $\mathbb{Z}^{d+1} / \mathbb{Z} \cdot(1, \ldots, 1)$;

(b) a $k+1$-tuple $\left(x_{0}, \ldots, x_{k}\right)$ of vertices in $A$ is a pointed $k$-simplex if and only if there is a sequence

$$
\emptyset \neq J_{0} \subsetneq \cdots \subsetneq J_{k-1} \subsetneq\{0, \ldots, d\}
$$

such that $\sum_{j \in J_{t}} e_{j}$ represents $x_{t}-x_{k}$ (formed with respect to the obvious group structure on $\left.\mathbb{Z}^{d+1} / \mathbb{Z} \cdot(1, \ldots, 1)\right)$, for any $0 \leqslant t \leqslant k-1$.

If $\left(x_{0}, \ldots, x_{k}\right)$ is a pointed $k$-simplex, we define $\ell\left(\left(x_{0}, \ldots, x_{k}\right)\right)$ to be the maximal number $r$ such that there exists a pointed $r$-simplex $\left(y_{0}, \ldots, y_{r}\right)$ with $x_{0}=y_{0}$ and $x_{k}=y_{r}$. For a pointed $(k-1)$-simplex $\widehat{\eta}=\left(x_{1}, \ldots, x_{k}\right)$ we define the set

$$
N_{\widehat{\eta}}=\left\{z \in X^{0} \mid\left(z, x_{1}, \ldots, x_{k}\right) \text { is a pointed } k \text {-simplex }\right\} .
$$

For $z \in N_{\widehat{\eta}}$ we write $(z, \widehat{\eta})$ for the pointed $k$-simplex $\left(z, x_{1}, \ldots, x_{k}\right)$. We define a partial ordering $\leqslant$ on $N_{\widehat{\eta}}$ by

$$
u_{1} \leqslant u_{2} \Longleftrightarrow\left[u_{1}=u_{2} \text { or }\left(u_{1}, u_{2}, \widehat{\eta}\right) \text { is a pointed }(k+1) \text {-simplex }\right] .
$$

Lemma 1.1. For any $u_{1}, u_{2} \in N_{\widehat{\eta}}$ the set

$$
W_{u_{1}, u_{2}}^{\widehat{\gamma}}=\left\{u \in N_{\widehat{\eta}} \mid u \leqslant u_{1} \text { and } u \leqslant u_{2}\right\}
$$

is empty or it contains an element $u$ such that $\ell((u, \widehat{\eta}))<\ell((w, \widehat{\eta}))$ for all $w \in W_{u_{1}, u_{2}}^{\widehat{\eta}}-\{u\}$.

Proof. Suppose we have two such candidates $u, u^{\prime}$. We can find an apartment $A$ which contains $\eta, u_{1}, u_{2}, u$ and $u^{\prime}$ (for example, because $\eta \cup\left\{u_{1}, u\right\}$ and $\left\{u^{\prime}, u_{2}\right\}$ are simplices). We identify $A$ with $\mathbb{R}^{d+1} / \mathbb{R} \cdot(1, \ldots, 1)$ as above. There exist subsets $\emptyset \neq J_{t} \subsetneq\{0, \ldots, d\}$ for $1 \leqslant t \leqslant k-1$ and $\emptyset \neq I_{s} \subsetneq\{0, \ldots, d\}$ for $s=1,2$ such that $x_{t}-x_{k}$ is represented by $\sum_{j \in J_{t}} e_{j}$ and $u_{s}-x_{k}$ is represented by $\sum_{j \in I_{s}} e_{j}$. We have $I_{s} \subsetneq J_{1} \subsetneq \cdots \subsetneq J_{k-1}$ for $s=1,2$. Hence, both $u-x_{k}$ and $u^{\prime}-x_{k}$ are represented by $\sum_{j \in I_{1} \cap I_{2}} e_{j}$.

If $W_{u_{1}, u_{2}}^{\widehat{\eta}} \neq \emptyset$ we denote the element $u \in W_{u_{1}, u_{2}}^{\widehat{\eta}}$ from Lemma 1.1 by $\left[\eta \mid u_{1}, u_{2}\right]$. If $W_{u_{1}, u_{2}}^{\widehat{\eta}}=\emptyset$ then $\left[\eta \mid u_{1}, u_{2}\right]$ is undefined. A subset $M_{0}$ of $N_{\widehat{\eta}}$ is called stable with respect to $\widehat{\eta}$ if for any two vertices $u_{1}, u_{2} \in M_{0}$ the vertex $\left[\widehat{\eta} \mid u_{1}, u_{2}\right]$ is defined and belongs to $M_{0}$. (See Lemma 2.2 below for what this means on Bruhat-Tits buildings. When working out the applications described later, we became fully convinced that stability is a very natural condition.) Note that $M_{0}$ is stable with respect 


\section{E. Grosse-KLÖNNE}

to $\widehat{\eta}=\left(x_{1}, \ldots, x_{k}\right)$ if and only if it is stable with respect to the pointed 0 -simplex $x_{k}$; this is because of $\left[x_{k} \mid u_{1}, u_{2}\right]=\left[\eta \mid u_{1}, u_{2}\right]$, as we saw in the proof of Lemma 1.1 .

A CCS $\mathcal{F}$ on $X$ is the assignment of an abelian group $\mathcal{F}(\tau)$ to every simplex $\tau$ of $X$, and a homomorphism $r_{\sigma}^{\tau}: \mathcal{F}(\tau) \rightarrow \mathcal{F}(\sigma)$ to every face inclusion $\tau \subset \sigma$, such that $r_{\rho}^{\sigma} \circ r_{\sigma}^{\tau}=r_{\rho}^{\tau}$ whenever $\tau \subset \sigma \subset \rho$, and $r_{\tau}^{\tau}$ is the identity.

Given a CCS $\mathcal{F}$, define the group $C^{k}(X, \mathcal{F})$ of $k$-cochains $(0 \leqslant k \leqslant d)$ to consist of the maps $c$, assigning to each $k$-simplex $\tau$ an element $c_{\tau} \in \mathcal{F}(\tau)$. Define

$$
\partial=\partial^{k+1}: C^{k}(X, \mathcal{F}) \longrightarrow C^{k+1}(X, \mathcal{F})
$$

by the rule

$$
(\partial c)_{\tau}=\sum_{\tau^{\prime} \subset \tau}\left[\tau: \tau^{\prime}\right] r_{\tau}^{\tau^{\prime}}\left(c_{\tau_{i}}\right)
$$

where $\left[\tau: \tau^{\prime}\right]= \pm 1$ is the incidence number (with respect to a fixed labeling of $X$ as in [Bro89, p. 30]). Then $\left(C^{\bullet}(X, \mathcal{F}), \partial\right)$ is a complex $\left(\partial^{2}=0\right)$, and its cohomology groups are denoted $H^{k}(X, \mathcal{F})$.

Consider for $1 \leqslant k \leqslant d$ the following condition $\mathcal{S}(k)$ for a CCS $\mathcal{F}$ on $X$ : for any pointed $(k-1)$ simplex $\widehat{\eta}$ with underlying $(k-1)$-simplex $\eta$ and for any subset $M_{0}$ of $N_{\widehat{\eta}}$ which is stable with respect to $\widehat{\eta}$, the following subquotient complex of $C^{\bullet}(X, \mathcal{F})$ is exact:

$$
\mathcal{F}(\eta) \stackrel{\partial^{k}}{\longrightarrow} \prod_{z \in M_{0}} \mathcal{F}(\{z\} \cup \eta) \stackrel{\partial^{k+1}}{\longrightarrow} \prod_{\substack{z, z^{\prime} \in M_{0} \\\left\{z, z^{\prime}\right\} \in X^{1}}} \mathcal{F}\left(\left\{z, z^{\prime}\right\} \cup \eta\right) .
$$

(We regard the first term as a subgroup of $C^{k-1}(X, \mathcal{F})$, the second as a direct summand of $C^{k}(X, \mathcal{F})$, and the third term as a quotient of $C^{k+1}(X, \mathcal{F})$.) Note that $\mathcal{S}(k)$ depends on the chosen orientation of $X$.

Theorem 1.2. Let $\mathcal{F}$ be a CCS on $X$. Let $1 \leqslant k \leqslant d$ and suppose $\mathcal{S}(k)$ holds true. Then $H^{k}(X, \mathcal{F})=0$.

We fix once and for all a vertex $z_{0} \in X^{0}$. Given an arbitrary vertex $x \in X^{0}$, choose an apartment $A$ containing $z_{0}$ and $x$. Choose an identification of $A$ with $\mathbb{R}^{d+1} / \mathbb{R} \cdot(1, \ldots, 1)$ as before, but now require in addition that $z_{0} \in A$ corresponds to the class of the origin in $\mathbb{R}^{d+1} / \mathbb{R} \cdot(1, \ldots, 1)$. Let $\sum_{j=0}^{d} m_{j} e_{j}$ be the unique representative of $x$ for which $m_{j} \geqslant 0$ for all $j$, and $m_{j}=0$ for at least one $j$. Let $\pi$ be a permutation of $\{0, \ldots, d\}$ such that $0=m_{\pi(d)} \geqslant \cdots \geqslant m_{\pi(0)}$ and set

$$
i(x)=\left(m_{\pi(d)}, \ldots, m_{\pi(1)}\right) \in \mathbb{N}_{0}^{d} .
$$

Lemma 1.3. The $d$-tuple $i(x)$ is independent of the choice of $A$.

Proof. Let us write $i_{A}(x)$ instead of $i(x)$ in order to indicate the reference to $A$ in the above definition. Suppose the apartment $A^{\prime}$ also contains $z_{0}$ and $x$. Choose a chamber $(d$-simplex) $C$ in $A$ containing $x$, and a chamber $C^{\prime}$ in $A^{\prime}$ containing $z_{0}$. Choose an apartment $A^{\prime \prime}$ in $X$ containing $C$ and $C^{\prime}$, and let $\pi: X \rightarrow A^{\prime \prime}$, respectively $\pi^{\prime}: X \rightarrow A^{\prime \prime}$, be the retraction from $X$ to $A^{\prime \prime}$ centered in $C$, respectively centered in $C^{\prime}$ (see [Bro89, p. 86]). Then $\pi$, respectively $\pi^{\prime}$, induces an isomorphism of oriented chamber complexes $A \cong A^{\prime \prime}$, respectively $A^{\prime} \cong A^{\prime \prime}$. Hence $i_{A}(x)=i_{A^{\prime \prime}}(x)=i_{A^{\prime}}(x)$.

Here is another, equivalent but more intrinsic definition of $i(x)$ (we do not need it). For $x, y \in X^{0}$ let $d(x, y) \in \mathbb{Z}_{\geqslant 0}$ be the minimal number $t$ such that there exists a sequence $x_{0}, \ldots, x_{t}$ in $X^{0}$ with $x=x_{0}, y=x_{t}$ and $\left\{x_{r-1}, x_{r}\right\} \in X^{1}$ for all $1 \leqslant r \leqslant t$. For $x \in X^{0}$ and a subset $W \subset X^{0}$ let $d(x, W)=\min \{d(x, y) \mid y \in W\}$. For $1 \leqslant i \leqslant d$ define the subset $W_{i}$ of $X^{0}$ inductively as follows: $W_{1}=\left\{z_{0}\right\}$ and

$$
W_{i}=\left\{z \in X^{0} \mid\left\{\begin{array}{c}
\text { there exist elements } z_{0}, \ldots, z_{r} \in X^{0} \text { such that } r=d\left(z, W_{i-1}\right), \\
z_{0} \in W_{i-1}, z_{r}=z \text { and } \ell\left(\left(z_{\ell-1}, z_{\ell}\right)\right)=1 \text { for all } 1 \leqslant \ell \leqslant r
\end{array}\right\}\right\} .
$$




\section{ACYCLIC COEFFICIENT SYSTEMS ON BUILDINGS}

In particular $W_{1} \subset W_{2} \subset \cdots \subset W_{d}$. For $x \in X^{0}$ we then have

$$
i(x)=\left(d\left(x, W_{1}\right), d\left(x, W_{2}\right), \ldots, d\left(x, W_{d}\right)\right) .
$$

Yet another equivalent definition of $i(x)$ (which we do not need either) results from the fact that the type of a minimal chamber-gallery connecting $x$ and $z_{0}$ encodes $i(x)$ if $x$ and $z_{0}$ are not incident.

On the set of ordered $d$-tuples $\left(n_{1}, \ldots, n_{d}\right) \in \mathbb{Z}_{\geqslant 0}^{d}$ (and hence on the set of $d$-tuples $i(x)$ for $\left.x \in X^{0}\right)$ we use the lexicographical ordering:

$$
\left(n_{1}, \ldots, n_{d}\right)<\left(n_{1}^{\prime}, \ldots, n_{d}^{\prime}\right) \Longleftrightarrow\left\{\begin{array}{c}
\text { there is a } 1 \leqslant r \leqslant d \text { such that } \\
n_{j}=n_{j}^{\prime} \text { for } 1 \leqslant j \leqslant r-1 \text { and } n_{r}<n_{r}^{\prime}
\end{array}\right\} .
$$

LEMma 1.4. Let $\eta$ be a $(k-1)$-simplex and let $x_{1}, \ldots, x_{k}$ be an enumeration of its vertices which satisfies $i\left(x_{1}\right) \leqslant \cdots \leqslant i\left(x_{k}\right)$. Then in fact $i\left(x_{1}\right)<\cdots<i\left(x_{k}\right)$ and $\widehat{\eta}=\left(x_{1}, \ldots, x_{k}\right)$ is a pointed $(k-1)$-simplex.

Proof. Choose an apartment $A$ containing $z_{0}$ and $\eta$, and choose an identification of $A$ with $\mathbb{R}^{d+1} / \mathbb{R}$. $(1, \ldots, 1)$ as before, with $z_{0} \in A$ corresponding to the class of the origin in $\mathbb{R}^{d+1} / \mathbb{R} \cdot(1, \ldots, 1)$. Then the claims follow easily from our description of the simplicial structure of $\mathbb{Z}^{d+1} / \mathbb{Z} \cdot(1, \ldots, 1)$.

Lemma 1.5. For any $x \in X^{0}, x \neq z_{0}$, there is among the vertices incident to $x$ a unique vertex $\nu(x)$ with minimal $i$-value: for all other vertices $z$ incident to $x$ we have $i(\nu(x))<i(z)$. If $z$ is incident to $x$, different from $\nu(x)$ and satisfies $i(z)<i(x)$, then $\nu(x)$ and $z$ are incident and $\ell((\nu(x), z)) \leqslant \ell((x, z))$.

Proof. Let $\left[z_{0}, x\right]$ be the geodesic (with respect to the Euclidean distance function on the geometric realization $|X|$ of $X$ ) between $z_{0}$ and $x$. Let $\tau$ be the minimal simplex which contains $x$ and whose open interior (viewed as a subset of $|X|$ ) contains a point of $\left[z_{0}, x\right]$. We assert that the vertex $\nu(x)$ of $\tau$ with minimal $i$-value is as claimed. To see this, let $A$ be an arbitrary apartment containing $x$ and $z_{0}$. Then $\tau$ is contained in $A$ because this is true for $\left[z_{0}, x\right]$. Explicitly it can be described as follows. Choose an identification of $A$ with $\mathbb{R}^{d+1} / \mathbb{R} \cdot(1, \ldots, 1)$ as before, with $z_{0} \in A$ corresponding to the class of the origin in $\mathbb{R}^{d+1} / \mathbb{R} \cdot(1, \ldots, 1)$. After reindexing the basis if necessary there are sequences $0 \leqslant r_{0}<r_{1}<\cdots<r_{s}=d$ and $0<m_{1}<\cdots<m_{s}$ (some $1 \leqslant s \leqslant d$ ) such that the vertex $x$ is represented by $y_{s}=\sum_{i=1}^{s} \sum_{j=r_{i-1}+1}^{r_{i}} m_{i} e_{j}$. Then $\left\{h y_{s} \mid 0 \leqslant h \leqslant 1\right\}$ represents $\left[z_{0}, x\right]$, and $\tau$ is a $s$-simplex, the other vertices are represented by $y_{t}=\sum_{i=1}^{t} \sum_{j=r_{i-1}+1}^{r_{i}} m_{i} e_{j}+\sum_{i=t+1}^{s} \sum_{j=r_{i-1}+1}^{r_{i}}$ $\left(m_{i}-1\right) e_{j}$ for $t=0, \ldots, s-1$. In particular, $\nu(x)$ is represented by $y_{0}$ and it is clear that it has minimal $i$-value among the vertices of $A$ incident to $x$. Since any vertex in $X$ incident to $x$ lies in such an $A$ the assertion follows. Also the other claims can immediately be read off from this analysis on an apartment.

Proposition 1.6. Let $\widehat{\eta}=\left(x_{1}, \ldots, x_{k}\right)$ be as in Lemma 1.4 and let $\eta=\left\{x_{1}, \ldots, x_{k}\right\}$. Then

$$
M_{0}=\left\{u \in X^{0} \mid\{u\} \cup \eta \text { is a } k \text {-simplex and } i(u)<i\left(x_{1}\right)\right\}
$$

is contained in $N_{\widehat{\eta}}$ and stable with respect to $\widehat{\eta}$.

Proof. The containment $M_{0} \subset N_{\widehat{\eta}}$ follows from Lemma 1.4 with $k$ instead of $(k-1)$. To prove that $M_{0}$ is stable with respect to $\widehat{\eta}$ let $u_{1}, u_{2} \in M_{0}$. First it follows from Lemma 1.4 (with $k$ and $k+1$ instead of $(k-1))$ and Lemma 1.5 that $\nu\left(x_{k}\right) \in W_{u_{1}, u_{2}}^{\widehat{\eta}}$, hence $\left[\widehat{\eta} \mid u_{1}, u_{2}\right]$ is defined. Since $\left(\left[\widehat{\eta} \mid u_{1}, u_{2}\right], x_{1}, \ldots, x_{k}\right)$ is a pointed $k$-simplex and since for any pointed $k$-simplex the underlying cyclic ordering of the vertices is independent of the pointing, there are, in view of Lemma 1.4 with $k$ instead of $(k-1)$, only the two possibilities $i\left(\left[\widehat{\eta} \mid u_{1}, u_{2}\right]\right)<i\left(x_{1}\right)$ and $i\left(\left[\widehat{\eta} \mid u_{1}, u_{2}\right]\right)>i\left(x_{k}\right)$. If $i\left(\left[\widehat{\eta} \mid u_{1}, u_{2}\right]\right)<i\left(x_{1}\right)$ then $\left[\widehat{\eta} \mid u_{1}, u_{2}\right] \in M_{0}$ and we are done. If $i\left(\left[\widehat{\eta} \mid u_{1}, u_{2}\right]\right)>i\left(x_{k}\right)$ then $\nu\left(\left[\widehat{\eta} \mid u_{1}, u_{2}\right]\right) \in W_{u_{1}, u_{2}}^{\widehat{\jmath}}$ by Lemmas 1.4 and 1.5. Moreover

$$
\ell\left(\left(\nu\left(\left[\widehat{\eta} \mid u_{1}, u_{2}\right]\right), \widehat{\eta}\right)\right) \leqslant \ell\left(\left(\left[\widehat{\eta} \mid u_{1}, u_{2}\right], \widehat{\eta}\right)\right)
$$




\section{E. Grosse-KLÖNnE}

also by Lemma 1.5. Since $\nu\left(\left[\widehat{\eta} \mid u_{1}, u_{2}\right]\right) \neq\left[\widehat{\eta} \mid u_{1}, u_{2}\right]$ this contradicts the definition of $\left[\widehat{\eta} \mid u_{1}, u_{2}\right]$. Hence $i\left(\left[\widehat{\eta} \mid u_{1}, u_{2}\right]\right)>i\left(x_{k}\right)$ cannot happen and the proof is finished.

Proof of Theorem 1.2. Given $\eta, \eta^{\prime} \in X^{k-1}$ let $x_{1}, \ldots, x_{k}$, respectively $x_{1}^{\prime}, \ldots, x_{k}^{\prime}$, be that enumeration of the vertices of $\eta$, respectively of $\eta^{\prime}$, which satisfies $i\left(x_{1}\right)<\cdots<i\left(x_{k}\right)$, respectively $i\left(x_{1}^{\prime}\right)<\cdots<i\left(x_{k}^{\prime}\right)$. We define

$$
\eta \stackrel{\sim}{<} \eta^{\prime} \Longleftrightarrow\left\{\begin{array}{c}
\text { there is a } 1 \leqslant q \leqslant k \text { such that } \\
i\left(x_{t}\right)=i\left(x_{t}^{\prime}\right) \text { for } 1 \leqslant t \leqslant q-1 \text { and } i\left(x_{q}\right)<i\left(x_{q}^{\prime}\right)
\end{array}\right\} .
$$

We define $\eta \cong \eta^{\prime}$ if $i\left(x_{t}\right)=i\left(x_{t}^{\prime}\right)$ for all $1 \leqslant t \leqslant k$. Let $\nabla: X^{k-1} \longrightarrow \mathbb{N}$ be the surjective map with

$$
\begin{aligned}
& \nabla(\eta)<\nabla\left(\eta^{\prime}\right) \Longleftrightarrow \eta \stackrel{\sim}{<} \eta^{\prime} \\
& \nabla(\eta)=\nabla\left(\eta^{\prime}\right) \Longleftrightarrow \eta \cong \eta^{\prime} .
\end{aligned}
$$

For a $k$-simplex $\sigma \in X^{k}$ let $\sigma^{-} \in X^{k-1}$ be the $(k-1)$-simplex obtained from $\sigma$ by omitting the vertex $x \in \sigma$ for which $i(x)$ is minimal. We need to show that

$$
\prod_{\eta \in X^{k-1}} \mathcal{F}(\eta) \stackrel{\partial^{k}}{\longrightarrow} \prod_{\sigma \in X^{k}} \mathcal{F}(\sigma) \stackrel{\partial^{k+1}}{\longrightarrow} \prod_{\tau \in X^{k+1}} \mathcal{F}(\tau)
$$

is exact. So let a $k$-cocycle $c=\left(c_{\sigma}\right)_{\sigma \in X^{k}} \in \operatorname{Ker}\left(\partial^{k+1}\right)$ be given. It suffices to show that there is a sequence of $(k-1)$-cochains $\left(b_{n}\right)_{n \in \mathbb{N}}=\left(\left(b_{n, \eta}\right)_{\eta \in X^{k-1}}\right)_{n \in \mathbb{N}}$ with $b_{n, \eta} \in \mathcal{F}(\eta)$ satisfying the following properties:

(i) $b_{n, \eta}=b_{\nabla(\eta), \eta}$ for all $n \geqslant \nabla(\eta)$;

(ii) $b_{n, \eta}=0$ for all $\eta \in X^{k-1}$ with $\nabla(\eta)>n$;

(iii) for all $\sigma \in X^{k}$ with $\nabla\left(\sigma^{-}\right) \leqslant n$ we have $\left(\partial^{k} b_{n}-c\right)_{\sigma}=0$.

Then the cochain $b_{\infty}=\left(b_{\infty, \eta}\right)_{\eta \in X^{k-1}}$ defined by $b_{\infty, \eta}=b_{\nabla(\eta), \eta}$ will be a preimage of $c$, as follows from (i) and (iii).

We construct $\left(b_{n}\right)_{n \in \mathbb{N}}$ inductively. Suppose $b_{n-1}$ has been constructed. We set $b_{n, \eta}=b_{n-1, \eta}$ for all $\eta \in X^{k-1}$ with $\nabla(\eta)<n$, and $b_{n, \eta}=0$ for all $\eta \in X^{k-1}$ with $\nabla(\eta)>n$. Now suppose we have $\eta \in X^{k-1}$ with $\nabla(\eta)=n$. Let $x_{1}, \ldots, x_{k}$ be that enumeration of the vertices of $\eta$ which satisfies $i\left(x_{1}\right)<\cdots<i\left(x_{k}\right)$. Consider the set

$$
M_{0}=\left\{z \in X^{0} \mid\{z\} \cup \eta \text { is a } k \text {-simplex and } i(z)<i\left(x_{1}\right)\right\} .
$$

We know from Lemma 1.4 and Proposition 1.6 that $\widehat{\eta}=\left(x_{1}, \ldots, x_{k}\right)$ is a pointed $(k-1)$-simplex and that $M_{0}$ is stable with respect to $\widehat{\eta}$ and contained in $N_{\widehat{\eta}}$. If $M_{0}=\emptyset$ we put $b_{n, \eta}=0$. So assume now that $M_{0} \neq \emptyset$. Let $z, z^{\prime} \in M_{0}$ with $\left\{z, z^{\prime}\right\} \in X^{1}$. We compute (with \pm , respectively $r$, denoting the respective incidence numbers, respectively restriction maps):

$$
\begin{aligned}
\partial^{k+1} & \left(\left(\left(\partial^{k} b_{n-1}-c\right)_{z^{\prime \prime} \cup \eta}\right)_{z^{\prime \prime} \in M_{0}}\right)_{\left.\left\{z, z^{\prime}\right\} \cup \eta\right\}} \\
& = \pm r\left(\left(\partial^{k} b_{n-1}-c\right)_{z \cup \eta}\right)+ \pm r\left(\left(\partial^{k} b_{n-1}-c\right)_{z^{\prime} \cup \eta}\right) \\
& = \pm r\left(\left(\partial^{k} b_{n-1}-c\right)_{z \cup \eta}\right)+ \pm r\left(\left(\partial^{k} b_{n-1}-c\right)_{z^{\prime} \cup \eta}\right)+\sum_{\left\{z, z^{\prime}\right\} \subset \sigma \subset\left\{z, z^{\prime}\right\} \cup \eta} \pm r\left(\left(\partial^{k} b_{n-1}-c\right)_{\sigma}\right) \\
& =\sum_{\sigma \subset\left\{z, z^{\prime}\right\} \cup \eta} \pm r\left(\left(\partial^{k} b_{n-1}-c\right)_{\sigma}\right) \\
& =\left(\partial^{k+1}\left(\partial^{k} b_{n-1}-c\right)\right)_{\left\{z, z^{\prime}\right\} \cup \eta}
\end{aligned}
$$

and this is zero because $c$ is a cocycle. For the second equality note that for all $\sigma \in X^{k}$ with $\left\{z, z^{\prime}\right\} \subset$ $\sigma \subset\left\{z, z^{\prime}\right\} \cup \eta$ we have $\nabla\left(\sigma^{-}\right)<n$ which by the induction hypothesis implies $\left(\partial^{k} b_{n-1}-c\right)_{\sigma}=0$. 
We have seen that $\left(\left(\partial^{k} b_{n-1}-c\right)_{\{z\} \cup \eta}\right)_{z \in M_{0}}$ lies in

$$
\operatorname{Ker}\left[\prod_{z \in M_{0}} \mathcal{F}(\{z\} \cup \eta) \stackrel{\partial^{k+1}}{\longrightarrow} \prod_{\substack{z, z^{\prime} \in M_{0} \\\left\{z, z^{\prime}\right\} \in X^{1}}} \mathcal{F}\left(\left\{z, z^{\prime}\right\} \cup \eta\right)\right] .
$$

We can therefore define $b_{n, \eta} \in \mathcal{F}(\eta)$ as a preimage of $\left[\sigma: \sigma^{-}\right]\left(\left(c-\partial^{k} b_{n-1}\right)_{\{z\} \cup \eta}\right)_{z \in M_{0}}$, by hypothesis $\mathcal{S}(k)$. To see that $b_{n}$ satisfies (iii) for $\sigma \in X^{k}$ with $\nabla\left(\sigma^{-}\right)=n$ we compute

$$
\begin{aligned}
\left(\partial b_{n}-c\right)_{\sigma} & =\left(\sum_{\eta \subset \sigma}[\sigma: \eta] r_{\sigma}^{\eta}\left(b_{n, \eta}\right)\right)-c_{\sigma} \\
& =\left(\sum_{\substack{\eta \subset \sigma \\
\nabla(\eta)<n}}[\sigma: \eta] r_{\sigma}^{\eta}\left(b_{n, \eta}\right)\right)+\left[\sigma: \sigma^{-}\right] r_{\sigma}^{\sigma^{-}}\left(b_{n, \sigma^{-}}\right)-c_{\sigma} \\
& =\left(\sum_{\substack{\eta \subset \sigma \\
\nabla(\eta)<n}}[\sigma: \eta] r_{\sigma}^{\eta}\left(b_{n-1, \eta}\right)\right)+\left(c-\partial^{k} b_{n-1}\right)_{\sigma}-c_{\sigma} \\
& =\left(\sum_{\substack{\eta \subset \sigma \\
\nabla(\eta)<n}}[\sigma: \eta] r_{\sigma}^{\eta}\left(b_{n-1, \eta}\right)\right)-\left(\sum_{\eta \subset \sigma}[\sigma: \eta] r_{\sigma}^{\eta}\left(b_{n-1, \eta}\right)\right)
\end{aligned}
$$

and this is zero because we have $b_{n-1, \sigma^{-}}=0$ by induction hypothesis (ii).

The reader will have observed that any $\mathbb{N}$-valued function $i$ on $X^{0}$ which takes different values on incident vertices gives rise to a local vanishing criterion like $\mathcal{S}(k)$, by the same formal proof above. However, the applicability of the resulting criterion depends on the control one gets over the corresponding sets $M_{0}$ defined analogously through formula (1). In this optic, the virtue of our particular choice of $i$ lies in the fact that we can control the corresponding sets $M_{0}$ : they satisfy the local (no reference to the global function $i$ ) property of being stable with respect to $\widehat{\eta}$; hence our vanishing criterion $\mathcal{S}(k)$, expressed entirely in local terms.

A HCS $\mathcal{V}$ of abelian groups on a building $X$ is the assignment of an abelian group $\mathcal{V}(\tau)$ to every simplex $\tau$ of $X$, and a homomorphism $r_{\sigma}^{\tau}: \mathcal{V}(\tau) \rightarrow \mathcal{V}(\sigma)$ to every face inclusion $\sigma \subset \tau$, such that $r_{\rho}^{\tau} \circ r_{\tau}^{\sigma}=r_{\rho}^{\sigma}$ whenever $\rho \subset \tau \subset \sigma$, and $r_{\tau}^{\tau}$ is the identity.

The group $C_{k}(X, \mathcal{V})$ of $k$-chains $(0 \leqslant k \leqslant d)$ consists of all the finitely supported maps $c$ which assign to each $k$-simplex $\tau$ an element $c_{\tau} \in \mathcal{V}(\tau)$. Define

$$
\partial=\partial_{k}: C_{k+1}(X, \mathcal{V}) \longrightarrow C_{k}(X, \mathcal{V})
$$

by the rule

$$
(\partial c)_{\tau}=\sum_{\tau^{\prime} \supset \tau}\left[\tau^{\prime}: \tau\right] r_{\tau}^{\tau^{\prime}}\left(c_{\tau^{\prime}}\right)
$$

Then $(C \cdot(X, \mathcal{V}), \partial)$ is a complex $\left(\partial^{2}=0\right)$, and its homology groups are denoted $H_{k}(X, \mathcal{V})$.

Consider for $1 \leqslant k \leqslant d$ the following condition $\mathcal{S}^{*}(k)$ for a HCS $\mathcal{V}$ on $X$ : for any pointed $(k-1)$-simplex $\widehat{\eta}$ with underlying $(k-1)$-simplex $\eta$ and for any subset $M_{0}$ of $N_{\widehat{\eta}}$ which is stable with respect to $\widehat{\eta}$, the following subquotient complex of $(C \cdot(X, \mathcal{V}), \partial)$ is exact:

$$
\bigoplus_{\substack{z, z^{\prime} \in M_{0} \\\left\{z, z^{\prime}\right\} \in X^{1}}} \mathcal{V}\left(\left\{z, z^{\prime}\right\} \cup \eta\right) \stackrel{\partial_{k}}{\longrightarrow} \bigoplus_{z \in M_{0}} \mathcal{V}(\{z\} \cup \eta) \stackrel{\partial_{k-1}}{\longrightarrow} \mathcal{V}(\eta) .
$$

TheOrem 1.7. Let $\mathcal{V}$ be a HCS on $X$. Let $1 \leqslant k \leqslant d$ and suppose $\mathcal{S}^{*}(k)$ holds true. Then $H_{k}(X, \mathcal{V})=0$. 


\section{E. Grosse-KLÖNnE}

Proof. We need to show that

$$
\bigoplus_{\tau \in X^{k+1}} \mathcal{V}(\tau) \stackrel{\partial_{k}}{\longrightarrow} \bigoplus_{\sigma \in X^{k}} \mathcal{V}(\sigma) \stackrel{\partial_{k-1}}{\longrightarrow} \bigoplus_{\eta \in X^{k-1}} \mathcal{V}(\eta)
$$

is exact. We use notations from the proof of Theorem 1.2. For $n \in \mathbb{Z}_{\geqslant 0}$ and elements $c=\left(c_{\sigma}\right)_{\sigma} \in$ $\oplus_{\sigma \in X^{k}} \mathcal{V}(\sigma)$ consider the condition

$$
C(n) \text { : for all } \sigma \in X^{k} \text { with } \nabla\left(\sigma^{-}\right) \geqslant n \text { we have } c_{\sigma}=0 .
$$

Similarly as in the proof of Theorem 1.2 one shows by induction on $n$ : all elements $c \in \operatorname{Ker}\left(\partial_{k-1}\right)$ which satisfy $C(n)$ lie in $\operatorname{Im}\left(\partial_{k}\right)$. Indeed, given such an element $c$ one uses $\mathcal{S}^{*}(k)$ in order to modify $c$ by an element of $\operatorname{Im}\left(\partial_{k}\right)$ in such a way that it even satisfies $C(n-1)$, and then the induction hypothesis applies.

\section{2. $p$-adic hyperplane arrangements}

Let $K$ denote a non-archimedean locally compact field, $\mathcal{O}_{K}$ its ring of integers, $\pi \in \mathcal{O}_{K}$ a fixed prime element and $k$ the residue field. Let $X$ be the Bruhat-Tits building of $\mathrm{PGL}_{d+1} / K$; it has Coxeter data of type $\widetilde{A}_{d}$. A concrete description of $X$ is the following. A lattice in the $K$-vector space $K^{d+1}$ is a free $\mathcal{O}_{K}$-submodule of $K^{d+1}$ of rank $d+1$. Two lattices $L, L^{\prime}$ are called homothetic if $L^{\prime}=\lambda L$ for some $\lambda \in K^{\times}$. We denote the homothety class of $L$ by $[L]$. The set of vertices of $X$ is the set of the homothety classes of lattices (always in $K^{d+1}$ ). For a lattice chain

$$
\pi L_{k} \subsetneq L_{0} \subsetneq L_{1} \subsetneq \cdots \subsetneq L_{k}
$$

we declare $\left(\left[L_{0}\right], \ldots,\left[L_{k}\right]\right)$ to be a pointed $k$-simplex. This defines a simplicial structure with orientation.

The following definitions are due to de Shalit [deS01] (who takes $R=K$, $\operatorname{char}(K)=0$ below). Let $\mathcal{A}$ be a non empty subset of $\mathbb{P}\left(K^{d+1}\right)$. We view $\mathcal{A}$ as a set of lines in $K^{d+1}$, or hyperplanes in $\left(K^{d+1}\right)^{*}$. We write $e_{a}$ for the line represented by $a \in K^{d+1}-\{0\}$, so that $e_{\lambda a}=e_{a}$ for any $\lambda \in K^{\times}$.

Let $R$ be a commutative ring and let $\widetilde{E}$ be the free exterior algebra over $R$, on the set $\mathcal{A}$. It is graded and anti-commutative. There is a unique derivation $\delta: \widetilde{E} \rightarrow \widetilde{E}$, homogeneous of degree -1 , mapping each $e \in \mathcal{A}$ to 1 . It satisfies $\delta^{2}=0$ and

$$
\begin{gathered}
\delta\left(e_{0} \wedge \cdots \wedge e_{k}\right)=\sum_{i=0}^{k}(-1)^{i} e_{0} \wedge \cdots \wedge \widehat{e_{i}} \wedge \cdots \wedge e_{k} \\
\operatorname{Im}(\delta)=\operatorname{Ker}(\delta) .
\end{gathered}
$$

The subalgebra $E=\operatorname{Im}(\delta)=\operatorname{Ker}(\delta)$ of $\widetilde{E}$ is generated by all elements $e-e^{\prime}$ for $e, e^{\prime} \in \mathcal{A}$. There is an exact sequence

$$
0 \longrightarrow E \longrightarrow \stackrel{\delta}{\rightarrow} E[1] \longrightarrow 0
$$

and any $e \in \mathcal{A}$ supplies a splitting $E[1] \rightarrow \widetilde{E}, x \mapsto e \wedge x$.

Let $x \in X^{0}$ be a vertex. We say that an element $g \in \widetilde{E}$ is a standard generator of $I(x)$ if there are a lattice $L_{x}$ representing $x$ and elements $\left\{a_{0}, \ldots, a_{m}\right\}$ of $\mathcal{A} \cap L_{x}-\pi L_{x}$, linearly dependent modulo $\pi L_{x}$, such that $g=\delta\left(e_{a_{0}} \wedge \cdots \wedge e_{a_{m}}\right)$. We define the ideal $I(x)$ in $\widetilde{E}$ as that generated by the standard generators of $I(x)$. For an arbitrary simplex $\sigma$ we define the ideal $I(\sigma)=\sum_{x \in \sigma} I(x)$. We set

$$
\widetilde{A}(\sigma)=\frac{\widetilde{E}}{I(\sigma)}, \quad A(\sigma)=\frac{E}{E \cap I(\sigma)}
$$




\section{ACYCLIC COEFFICIENT SYSTEMS ON BUILDINGS}

The split exact sequence (2) provides us with a split exact sequence

$$
0 \longrightarrow A(\sigma) \longrightarrow \widetilde{A}(\sigma) \stackrel{\delta}{\rightarrow} A(\sigma)[1] \longrightarrow 0 .
$$

The ideal $I(\sigma)$ is homogeneous, hence there is a natural grading on $\widetilde{A}(\sigma)$ and on $A(\sigma)$. We denote by $\widetilde{A}^{q}(\sigma)$ respectively by $A^{q}(\sigma)$ the $q$ th graded piece. For varying $\sigma$ the $\widetilde{A}(\sigma)$ and $A(\sigma)$ form CCSs $\widetilde{A}$ and $A$ on $X$.

Suppose that we are given a lattice chain

$$
\pi L_{k} \subsetneq L_{1} \subsetneq L_{2} \subsetneq \cdots \subsetneq L_{k} .
$$

Let $x_{j} \in X^{0}$ be the vertex defined by $L_{j}$; then $\widehat{\eta}=\left(x_{1}, \ldots, x_{k}\right)$ is a pointed $(k-1)$-simplex; we denote by $\eta$ the underlying non pointed $(k-1)$-simplex. We write $L_{0}=\pi L_{k}$. As long as $L_{k}$ is fixed we abuse notation in that we identify an element $e \in \mathcal{A}$ with an element $a \in L_{k}-L_{0}$ for which $e=e_{a}$; such an $a$ is unique up to a unit in $\mathcal{O}_{K}$. Thus we regard $\mathcal{A}$ as a subset of $L_{k}-L_{0}$.

Assume that $\mathcal{A}$ is finite and fix a linear ordering $\prec$ on $\mathcal{A}$ which is adapted to $\widehat{\eta}$. By definition, this means $\max \left(\mathcal{A} \cap L_{j}-L_{j-1}\right) \prec \min \left(\mathcal{A} \cap L_{j+1}-L_{j}\right)$ for all $1 \leqslant j \leqslant k-1$. For $S \subset \mathcal{A}$ and $e \in S$ we define the $\mathcal{O}_{K}$-submodule $L(\widehat{\eta}, \prec, S, e)$ of $K^{d+1}$ as follows: let $1 \leqslant j \leqslant k$ be the number for which $e \in L_{j}-L_{j-1}$; then

$$
L(\widehat{\eta}, \prec, S, e)=\left\langle L_{j-1},\left\{e^{\prime} \in S \mid e^{\prime} \prec e \text { or } e^{\prime}=e\right\}\right\rangle_{\mathcal{O}_{K}} .
$$

We say that $e$ is $(S, \widehat{\eta})$-special with respect to $\prec$ if

$$
e=\max _{\prec}(\mathcal{A} \cap L(\widehat{\eta}, \prec, S, e)) .
$$

(Here $\max _{\prec}(Q)$ for a subset $Q$ of $\mathcal{A}$ means the maximal element of $Q$ with respect to the fixed ordering $\prec$. The subscript $\prec$ does not indicate that $\prec$ is a running parameter.)

Fix another linear ordering $<$ on $\mathcal{A}$ and for subsets $S$ of $\mathcal{A}$ let $e_{S}=e_{0} \wedge \cdots \wedge e_{r} \in \widehat{E}$ where $e_{0}<e_{1}<\cdots<e_{r}$ is the increasing enumeration of the elements of $S$ in the ordering $<$. (The ordering $<$ may be taken to be $\prec$, but the role of these two orderings will be completely unrelated in the following.)

Lemma 2.1. The free $R$-module $\widetilde{A}(\eta)$ is of finite rank, a basis is the set

$$
\left\{e_{S} \mid S \subset \mathcal{A} \text {, all } e \in S \text { are }(S, \widehat{\eta}) \text {-special with respect to } \prec\right\} \text {. }
$$

Proof. This is [deS01, Theorem 2.5]: the 'broken circuit theorem' (there $R=K, \operatorname{char}(K)=0$ and $\prec=<$, but this is irrelevant).

Let $N_{\widehat{\eta}}$ be as in $\S 1$. A subset $M_{0} \subset N_{\widehat{\eta}}$ corresponds to a collection of lattices $\left(L_{z}\right)_{z \in M_{0}}$ with $L_{0} \subsetneq L_{z} \subsetneq L_{1}$ for all $z \in M_{0}$. The following lemma is clear.

Lemma 2.2. We have $M_{0}$ is stable with respect to $\widehat{\eta}$ if and only if for all $z_{1}, z_{2} \in M_{0}$ the lattice $L_{z_{1}} \cap L_{z_{2}}$ represents an element of $M_{0}$.

Suppose that $M_{0} \subset N_{\widehat{\eta}}$ is stable with respect to $\widehat{\eta}$. In particular there is a $x_{0} \in M_{0}$ with $L_{x_{0}} \subset L_{z}$ for all $z \in M_{0}$. We say that a collection $\left(\prec_{z}\right)_{z \in M_{0}}$, indexed by $M_{0}$, of linear orderings on $\mathcal{A}$ is adapted to $\left(M_{0}, \widehat{\eta}\right)$ if the following conditions hold:

- for any $z \in M_{0}$ the ordering $\prec_{z}$ is adapted to the pointed $k$-simplex $(z, \widehat{\eta})$;

- for any $z_{1}, z_{2} \in M_{0}$ with $L_{z_{1}} \subset L_{z_{2}}$ we have $\left[e \prec_{z_{1}} e^{\prime} \Leftrightarrow e \prec_{z_{2}} e^{\prime}\right]$ for all pairs $e, e^{\prime} \in \mathcal{A} \cap L_{z_{1}}$ and also for all pairs $e, e^{\prime} \in \mathcal{A} \cap L_{z_{2}}-L_{z_{1}}$;

- for any $z \in M_{0}$ we have $\left[e \prec_{x_{0}} e^{\prime} \Leftrightarrow e \prec_{z} e^{\prime}\right]$ for all pairs $e, e^{\prime} \in \mathcal{A} \cap L_{k}-L_{z}$;

- we have $e \prec_{x_{0}} e^{\prime}$ for all pairs $e, e^{\prime} \in \mathcal{A}$ with $e \in \bigcup_{z \in M_{0}} L_{z}$ and $e^{\prime} \notin \bigcup_{z \in M_{0}} L_{z}$. 


\section{E. Grosse-KLÖNnE}

Lemma 2.3. Collections of linear orderings on $\mathcal{A}$ adapted to $\left(M_{0}, \widehat{\eta}\right)$ exist.

Proof. The referee suggested the following proof (our original was unnecessarily complicated). Let $U=\bigcup_{z \in M_{0}} L_{z}$. Fix a linear ordering $\prec$ on $\mathcal{A}$ adapted to $\widehat{\eta}$ which satisfies $e \prec e^{\prime}$ for all pairs $e, e^{\prime} \in \mathcal{A}$ with $e \in U$ and $e^{\prime} \notin U$. For a $z \in M_{0}$ let $\prec_{z}$ be the ordering which satisfies firstly $e \prec_{z} e^{\prime} \prec_{z} e^{\prime \prime}$ for all triples $e, e^{\prime}, e^{\prime \prime} \in \mathcal{A}$ with $e \in L_{z}$, with $e^{\prime} \in U-L_{z}$ and with $e^{\prime \prime} \in L_{k}-U$, and secondly $\left[e \prec e^{\prime} \Leftrightarrow e \prec_{z} e^{\prime}\right]$ for all pairs $e, e^{\prime} \in \mathcal{A} \cap L_{z}$, for all pairs $e, e^{\prime} \in \mathcal{A} \cap U-L_{z}$ and for all pairs $e, e^{\prime} \in \mathcal{A}-(\mathcal{A} \cap U)$. It is straightforwardly checked that $\left(\prec_{z}\right)_{z \in M_{0}}$ is adapted to $\left(M_{0}, \widehat{\eta}\right)$.

Fix a collection $\left(\prec_{z}\right)_{z \in M_{0}}$ of linear orderings on $\mathcal{A}$ adapted to $\left(M_{0}, \widehat{\eta}\right)$. Let

$$
G\left(\widehat{\eta} ; M_{0}\right)=\left\{\begin{array}{c|c}
e_{S} \mid \begin{array}{c}
S \subset \mathcal{A}, \text { for all } e \in S \text { there is a } \\
z \in M_{0} \text { such that } e \text { is }(S,(z, \widehat{\eta})) \text {-special }
\end{array}
\end{array}\right\}
$$

where $(S,(z, \widehat{\eta}))$-speciality is to be understood with respect to $\prec_{z}$. Let $J\left(\widehat{\eta} ; M_{0}\right) \subset \widetilde{E}$ be the ideal generated by the set

$$
I(\eta) \bigcup\left\{g \in \widetilde{E} \mid g \text { is a standard generator of } I(z) \text { for all } z \in M_{0}\right\} .
$$

Proposition 2.4. We have $G\left(\widehat{\eta} ; M_{0}\right)$ is finite and generates $\widetilde{E} / J\left(\widehat{\eta} ; M_{0}\right)$ as an $R$-module.

Proof. Clearly the set of all $e_{S}$ with $S \subset \mathcal{A}$ is generating. Now suppose that $S \subset \mathcal{A}$ does not satisfy the condition defining $G\left(\widehat{\eta} ; M_{0}\right)$. That is, there exists an $e \in S$ such that for all $z \in M_{0}$ this $e$ is not $(S,(z, \widehat{\eta}))$-special. Fix such an $e$.

We first claim that there is an $\widehat{e} \in S$ and a subset $\widehat{S} \subset S$ such that for all $z \in M_{0}$ the following statements (1) and (2) are satisfied:

(1) for all $e^{\prime} \in \widehat{S}$ we have $e^{\prime} \prec_{z} e \prec_{z} \widehat{e}$;

(2) The element $\delta\left(e_{\widehat{S} \cup\{e, \widehat{e}\}}\right)$ belongs to $J\left(\widehat{\eta} ; M_{0}\right)$.

We distinguish three cases. First consider the case $e \in L_{j}-L_{j-1}$ for some $2 \leqslant j \leqslant k$. Then we set $\widehat{S}=\left\{e^{\prime} \in S \mid e^{\prime} \prec_{x_{0}} e\right.$ and $\left.e^{\prime} \notin L_{j-1}\right\}$ and

$$
\widehat{e}=\max _{\prec_{0}}\left(\mathcal{A} \cap L\left(\left(x_{0}, \widehat{\eta}\right), \prec_{x_{0}}, S, e\right)\right) .
$$

The fact that $e$ is not $\left(S,\left(x_{0}, \widehat{\eta}\right)\right)$-special and the properties of the adapted collection $\left(\prec_{z}\right)_{z \in M_{0}}$ give statement (1). Moreover $\widehat{S} \cup\{e, \widehat{e}\}$ is linearly dependent modulo $L_{j-1}$, hence $\delta\left(e_{\widehat{S} \cup\{e, \widehat{e}\}}\right)$ lies in $I\left(x_{j-1}\right) \subset I(\eta)$ and we get statement $(2)$.

Now consider the case $e \in L_{1}-\bigcup_{z \in M_{0}} L_{z}$. Then we set $\widehat{S}=\left\{e^{\prime} \in S \mid e^{\prime} \prec_{x_{0}} e\right\}$ and $\widehat{e}=$ $\max _{\prec x_{0}}\left(\mathcal{A} \cap L\left(\left(x_{0}, \widehat{\eta}\right), \prec_{x_{0}}, S, e\right)\right)$. Again the fact that $e$ is not $\left(S,\left(x_{0}, \widehat{\eta}\right)\right)$-special and the properties of the adapted collection $\left(\prec_{z}\right)_{z \in M_{0}}$ give statement (1). For $z \in M_{0}$ let $\widehat{S}_{z}=\widehat{S}-\left(\widehat{S} \cap L_{z}\right)$. The fact that $e$ is not $(S,(z, \widehat{\eta}))$-special tells us that $\widehat{S}_{z} \cup\{e, \widehat{e}\}$ is also linearly dependent modulo $L_{z}$. However then the subset of $\pi^{-1} L_{z}-L_{z}$ which defines the same elements in $\mathbb{P}\left(K^{d+1}\right)$ as does $\widehat{S} \cup\{e, \widehat{e}\}$ is linearly dependent modulo $L_{z}$, because it contains $\widehat{S}_{z} \cup\{e, \widehat{e}\}$. Therefore, $\delta\left(e_{\widehat{S} \cup\{e, \widehat{e}\}}\right)$ is a standard generator of $I(z)$. We have shown statement (2).

Finally the case $e \in \bigcup_{z \in M_{0}} L_{z}$. Let $z_{e} \in M_{0}$ be such that $e \in L_{z_{e}}$ and $L_{z_{e}}$ is minimal with this property (this $z_{e}$ is unique since $M_{0}$ is stable). We set $\widehat{S}=\left\{e^{\prime} \in S \mid e^{\prime} \prec_{z_{e}} e\right\}$ and $\widehat{e}=$ $\max _{\prec_{z}}\left(\mathcal{A} \cap L\left(\left(z_{e}, \widehat{\eta}\right), \prec_{z_{e}}, S, e\right)\right)$. The fact that $e$ is not $\left(S,\left(z_{e}, \widehat{\eta}\right)\right)$-special gives statements $(1)$ and (2) in this case (here $\left.\delta\left(e_{\widehat{S} \cup\{e, \widehat{e}\}}\right) \in I\left(x_{k}\right) \subset I(\eta)\right)$. Again this is straightforwardly checked using the properties of the adapted collection $\left(\prec_{z}\right)_{z \in M_{0}}$.

The claim established, statement (2) tells us that we may replace $e_{\widehat{S} \cup\{e\}}$ in $\widetilde{E} / J\left(\widehat{\eta} ; M_{0}\right)$ by a linear combination of elements $e_{S^{\prime}}$ with each $S^{\prime}$ arising from $\widehat{S} \cup\{e, \widehat{e}\}$ by deleting an element of $\widehat{S} \cup\{e\}$. 


\section{ACYCLIC COEFFICIENT SYSTEMS ON BUILDINGS}

By statement (1) of our claim this means that we may replace $e_{S}$ by a linear combination of elements $e_{S^{\prime \prime}}$ with each $S^{\prime \prime}$ satisfying $S \prec_{z} S^{\prime \prime}$ for all $z \in M_{0}$ (for the lexicographic ordering $\prec_{z}$ on the set of subsets of $\mathcal{A}$ of fixed cardinality derived from the ordering $\prec_{z}$ on $\mathcal{A}$ ). Repeating the process proves that $G\left(\widehat{\eta} ; M_{0}\right)$ is generating. That it is finite follows from Lemma 2.1. We are done.

We define the complex

$$
K\left(\widehat{\eta}, M_{0}\right)=\left\{\begin{array}{c}
0 \longrightarrow \frac{\widetilde{E}}{\bigcap_{z \in M_{0}} I(\eta \cup\{z\})} \longrightarrow \\
\prod_{z \in M_{0}} \widetilde{A}(\eta \cup\{z\}) \longrightarrow \prod_{\substack{z_{1}, z_{2} \in M_{0} \\
\left\{z_{1}, z_{2}\right\} \in X^{1}}} \widetilde{A}\left(\eta \cup\left\{z_{1}, z_{2}\right\}\right) \longrightarrow \cdots
\end{array}\right\} .
$$

Proposition 2.5. For non-empty $M_{0}$ as above the following statements hold:

(a) the complex $K\left(\widehat{\eta}, M_{0}\right)$ is exact;

(b) $J\left(\widehat{\eta} ; M_{0}\right)=\bigcap_{z \in M_{0}} I(\eta \cup\{z\})$;

(c) $\widetilde{E} / \bigcap_{z \in M_{0}} I(\eta \cup\{z\})$ is a free $R$-module and $G\left(\widehat{\eta} ; M_{0}\right)$ is a basis.

Proof. For $n \geqslant 1$ let $(\mathrm{a})_{n},(\mathrm{~b})_{n}$ and $(\mathrm{c})_{n}$ be the corresponding statements for all $M_{0}$ with $1 \leqslant$ $\left|M_{0}\right| \leqslant n$. We will prove these statements by simultaneous induction on $n$.

Statements $(\mathrm{a})_{1}$ and $(\mathrm{b})_{1}$ are clear, and $(\mathrm{c})_{1}$ is Lemma 2.1. Now we assume $n>1$. First we prove (a) $)_{n}$. Choose a $y \in M_{0}$ for which $L_{y}$ is maximal, i.e. such that there is no $z \in M_{0}$ with $L_{y} \subsetneq L_{z}$. If we set

$$
M_{1}=M_{0}-\{y\}, \quad M_{1}^{\prime}=\left\{z \in M_{1} \mid L_{z} \subset L_{y}\right\}
$$

then $M_{1}$ and $M_{1}^{\prime}$ are stable with respect to $\widehat{\eta}$ respectively $(y, \widehat{\eta})$. We claim

$$
\bigcap_{z^{\prime} \in M_{1}^{\prime}} I\left(\eta \cup\left\{y, z^{\prime}\right\}\right) \subset I(\eta \cup\{y\})+\bigcap_{z \in M_{1}} I(z) .
$$

For $x \in M_{0}$ and $S \subset \mathcal{A}$ let $S^{x}$ be the uniquely determined subset of $L_{x}-\pi L_{x}$ which determines the same set of lines in $K^{d+1}$ (or the same subset of $\mathbb{P}\left(K^{d+1}\right)$ ) as does $S$ (recall that by convention we view $S$ as a subset of $L_{k}-L_{0}$ ). By induction hypothesis (b) ${ }_{n-1}$, applied to the pointed $k$-cell $(y, \widehat{\eta})$ and $M_{1}^{\prime} \subset N_{(y, \widehat{\eta})}$, a typical generator $g$ of the left-hand side of (4) satisfies at least one of the following conditions:

(i) $g \in I(\eta \cup\{y\})$;

(ii) $g=\delta\left(e_{0} \wedge \cdots \wedge e_{r}\right)$ for some $S=\left\{e_{0}, \ldots, e_{r}\right\} \subset \mathcal{A}$ such that $S^{z^{\prime}}$ is linearly dependent modulo $\pi L_{z^{\prime}}$ for all $z^{\prime} \in M_{1}^{\prime}$.

To show that $g$ lies in the right-hand side of (4) is difficult only if (i) does not hold. In that case we claim $g \in \bigcap_{z \in M_{1}} I(z)$. So let $S$ be as in (ii) and let $z \in M_{1}$ be given. Since $M_{0}$ is stable with respect to $\widehat{\eta}$ there exists a $z^{\prime} \in M_{1}^{\prime}$ such that $L_{y} \cap L_{z}=L_{z^{\prime}}$. Now $S^{z^{\prime}}$ is linearly dependent modulo $\pi L_{z^{\prime}}$. If $S^{y}$ was linearly dependent modulo $\pi L_{y}$ we would have $g \in I(y)$, but then (i) would hold. Hence $S^{y}$ is linearly independent modulo $\pi L_{y}$, hence so is $S^{z^{\prime}} \cap S^{y}$, and hence $\widetilde{S}=S^{z^{\prime}}-\left(S^{z^{\prime}} \cap S^{y}\right)$ is linearly dependent modulo $\pi L_{z}$ (since $S^{z^{\prime}}$ is). On the other hand $\widetilde{S} \subset \pi L_{y}$ and this implies $\widetilde{S} \cap \pi L_{z}=\emptyset$ (since $\pi L_{y} \cap \pi L_{z}=\pi L_{z^{\prime}}$ ). Thus, $\widetilde{S}$ is a subset of $L_{z}-\pi L_{z}$ which is linearly dependent modulo $\pi L_{z}$. In particular, $S^{z}$ is linearly dependent modulo $\pi L_{z}$ (since $\widetilde{S} \subset S^{z}$ ), hence $g \in I(z)$. 


\section{E. Grosse-KLÖNnE}

We have established the claim (4). Next we claim that

$$
\widetilde{E} \longrightarrow \prod_{z \in M_{0}} \widetilde{A}(\eta \cup\{z\}) \longrightarrow \prod_{\substack{z_{1}, z_{2} \in M_{0} \\\left\{z_{1}, z_{2}\right\} \in X^{1}}} \widetilde{A}\left(\eta \cup\left\{z_{1}, z_{2}\right\}\right)
$$

is exact; this is equivalent with exactness of $K\left(\widehat{\eta}, M_{0}\right)$ at the first non trivial degree. Let $\left(s_{z}\right)_{z \in M_{0}}$ be an element of the kernel of the second arrow in (5). By induction hypothesis (a) ${ }_{n-1}$ for $M_{1}$ we may assume, after modifying $\left(s_{z}\right)_{z \in M_{0}}$ by the image of an element of $\widetilde{E}$, that $s_{z}=0$ for all $z \in M_{1}$. Then it follows that

$$
s_{y} \in \operatorname{Ker}\left[\widetilde{A}(\eta \cup\{y\}) \longrightarrow \prod_{z^{\prime} \in M_{1}^{\prime}} \widetilde{A}\left(\eta \cup\left\{z^{\prime}, y\right\}\right)\right] .
$$

This means that $s_{y}$ can be lifted to an element $\tilde{s}_{y}$ of the left-hand side of (4). By (4) there exist $\tilde{b} \in I(\eta \cup\{y\})$ and $\tilde{c} \in \bigcap_{z \in M_{1}} I(\eta \cup\{z\})$ with $\tilde{s}_{y}=\tilde{b}+\tilde{c}$. Thus $\tilde{c}$ is a preimage of $\left(s_{z}\right)_{z \in M_{0}}$ and the exactness of $(5)$ is proven. If we define the complex

$$
K^{y}\left(\widehat{\eta}, M_{0}\right)=\left\{\begin{array}{c}
0 \longrightarrow \frac{\widetilde{E}}{\bigcap_{z \in M_{1}} I(\eta \cup\{z\})} \longrightarrow \\
\frac{\widetilde{E}}{\bigcap_{z^{\prime} \in M_{1}^{\prime}} I\left(\eta \cup\left\{y, z^{\prime}\right\}\right)} \times \prod_{z \in M_{1}} \widetilde{A}(\eta \cup\{z\}) \longrightarrow \\
\prod_{\substack{z_{1}, z_{2} \in M_{0} \\
\left\{z_{1}, z_{2}\right\} \in X^{1}}} \widetilde{A}\left(\eta \cup\left\{z_{1}, z_{2}\right\}\right) \longrightarrow \prod_{\substack{z_{1}, z_{2}, z_{3} \in M_{0} \\
\left\{z_{1}, z_{2}, z_{3}\right\} \in X^{2}}} \widetilde{A}\left(\eta \cup\left\{z_{1}, z_{2}, z_{3}\right\}\right) \longrightarrow \cdots
\end{array}\right\}
$$

then we have a short exact sequence

$$
0 \longrightarrow K\left((y, \widehat{\eta}), M_{1}^{\prime}\right)[-1] \longrightarrow K^{y}\left(\widehat{\eta}, M_{0}\right) \longrightarrow K\left(\widehat{\eta}, M_{1}\right) \longrightarrow 0 .
$$

By induction hypothesis the complexes $K\left((y, \widehat{\eta}), M_{1}^{\prime}\right)$ and $K\left(\widehat{\eta}, M_{1}\right)$ are exact; hence the complex $K^{y}\left(\widehat{\eta}, M_{0}\right)$ is exact. The exactness of $K^{y}\left(\widehat{\eta}, M_{0}\right)$ shows the exactness of $K\left(\widehat{\eta}, M_{0}\right)$ except at the first non-trivial degree, but at the first non-trivial degree we have already seen exactness. Hence $K\left(\widehat{\eta}, M_{0}\right)$ is exact and $(\mathrm{a})_{n}$ is proven.

If we define the complex

$$
N^{y}\left(\widehat{\eta}, M_{0}\right)=\left\{0 \longrightarrow \frac{\bigcap_{z \in M_{1}} I(\eta \cup\{z\})}{\bigcap_{z \in M_{0}} I(\eta \cup\{z\})} \longrightarrow \frac{\bigcap_{z \in M_{1}^{\prime}} I(\eta \cup\{y, z\})}{I(\eta \cup\{y\})} \longrightarrow 0 \longrightarrow \cdots\right\}
$$

then we have a short exact sequence

$$
0 \longrightarrow N^{y}\left(\widehat{\eta}, M_{0}\right) \longrightarrow K\left(\widehat{\eta}, M_{0}\right) \longrightarrow K^{y}\left(\widehat{\eta}, M_{0}\right) \longrightarrow 0 .
$$

Since we have seen exactness of $K\left(\widehat{\eta}, M_{0}\right)$ and of $K^{y}\left(\widehat{\eta}, M_{0}\right)$ we get exactness of $N^{y}\left(\widehat{\eta}, M_{0}\right)$. Now to prove $(\mathrm{b})_{n}$ and $(\mathrm{c})_{n}$ we first suppose $R=\mathbb{Z}$. By exactness of $N^{y}\left(\widehat{\eta}, M_{0}\right)$ we get

$$
\begin{aligned}
\operatorname{rank}_{\mathbb{Z}}\left(\frac{\widetilde{E}}{\bigcap_{z \in M_{0}} I(\eta \cup\{z\})}\right)-\operatorname{rank}_{\mathbb{Z}}\left(\frac{\widetilde{E}}{\bigcap_{z \in M_{1}} I(\eta \cup\{z\})}\right) \\
\quad=\operatorname{rank}_{\mathbb{Z}}(\widetilde{A}(\eta \cup\{y\}))-\operatorname{rank}_{\mathbb{Z}}\left(\frac{\widetilde{E}}{\bigcap_{z \in M_{1}^{\prime}} I(\eta \cup\{y, z\})}\right) .
\end{aligned}
$$

Observe that the collection $\left(\prec_{z}\right)_{z \in M_{1}}$, respectively $\left(\prec_{z^{\prime}}\right)_{z^{\prime} \in M_{1}^{\prime}}$, respectively $\prec_{y}$ is adapted to $\left(M_{1}, \widehat{\eta}\right)$, respectively to $\left(M_{1}^{\prime},(y, \widehat{\eta})\right)$, respectively $(\{y\}, \widehat{\eta})$. We associate the sets $G\left(\widehat{\eta} ; M_{1}\right)$, 


\section{ACYCLIC COEFFICIENT SYSTEMS ON BUILDINGS}

respectively $G\left((y, \widehat{\eta}) ; M_{1}^{\prime}\right)$, respectively $G(\widehat{\eta} ;\{y\})$ as before and claim

$$
\begin{gathered}
G\left(\widehat{\eta} ; M_{0}\right)=G\left(\widehat{\eta} ; M_{1}\right) \cup G(\widehat{\eta} ;\{y\}) \\
G\left((y, \widehat{\eta}) ; M_{1}^{\prime}\right)=G\left(\widehat{\eta} ; M_{1}\right) \cap G(\widehat{\eta} ;\{y\}) .
\end{gathered}
$$

Here (7) and $\subset$ in (8) are very easy. To prove $\supset$ in (8), let $e_{S} \in G\left(\widehat{\eta} ; M_{1}\right) \bigcap G(\widehat{\eta} ;\{y\})$ and let $e \in S$. Then $e$ is $(S,(y, \widehat{\eta}))$-special and $(S,(z, \widehat{\eta}))$-special for some $z \in M_{1}$. Let $z^{\prime} \in M_{1}^{\prime}$ be the element with $L_{y} \cap L_{z}=L_{z^{\prime}}$. We will show that $e$ is $(S,(z, y, \widehat{\eta}))$-special. If $e \in L_{y}$, then

$$
e=\max _{\prec z}\left(\mathcal{A} \cap L\left((z, \widehat{\eta}), \prec_{z}, S, e\right)\right)=\max _{\prec_{z^{\prime}}}\left(\mathcal{A} \cap L\left(\left(z^{\prime}, y, \widehat{\eta}\right), \prec_{z^{\prime}}, S, e\right)\right)
$$

where the first equality follows from the $(S,(z, \widehat{\eta}))$-speciality of $e$ and the second one from $L_{y} \cap L_{z}$ $=L_{z^{\prime}}$. If, however, $e \notin L_{y}$, then

$$
e=\max _{\prec_{y}}\left(\mathcal{A} \cap L\left((y, \widehat{\eta}), \prec_{y}, S, e\right)\right)=\max _{\prec_{z^{\prime}}}\left(\mathcal{A} \cap L\left(\left(z^{\prime}, y, \widehat{\eta}\right), \prec_{z^{\prime}}, S, e\right)\right)
$$

where the first equality follows from the $(S,(y, \widehat{\eta}))$-speciality of $e$ and the second one is clear.

From (7) and (8) we deduce

$$
\left|G\left(\widehat{\eta} ; M_{0}\right)\right|=\left|G\left(\widehat{\eta} ; M_{1}\right)\right|+|G(\widehat{\eta} ;\{y\})|-\left|G\left((y, \widehat{\eta}) ; M_{1}^{\prime}\right)\right| \text {. }
$$

By induction hypothesis $(c)_{n-1}$ we know

$$
\begin{aligned}
\left|G\left(\widehat{\eta} ; M_{1}\right)\right| & =\operatorname{rank}_{\mathbb{Z}}\left(\frac{\widetilde{E}}{\bigcap_{z \in M_{1}} I(\eta \cup\{z\})}\right) \\
|G(\widehat{\eta} ;\{y\})| & =\operatorname{rank}_{\mathbb{Z}}(\widetilde{A}(\eta \cup\{y\})) \\
\left|G\left((y, \widehat{\eta}) ; M_{1}^{\prime}\right)\right| & =\operatorname{rank}_{\mathbb{Z}}\left(\frac{\widetilde{E}}{\bigcap_{z \in M_{1}^{\prime}} I(\eta \cup\{y, z\})}\right) .
\end{aligned}
$$

Thus we may compare (6) with (9) to obtain

$$
\operatorname{rank}_{\mathbb{Z}}\left(\frac{\widetilde{E}}{\bigcap_{z \in M_{0}} I(\eta \cup\{z\})}\right)=\left|G\left(\widehat{\eta} ; M_{0}\right)\right| .
$$

Comparing with Proposition 2.4 we see that source and target of the canonical surjection

$$
\frac{\widetilde{E}}{J\left(\widehat{\eta} ; M_{0}\right)} \longrightarrow \frac{\widetilde{E}}{\bigcap_{z \in M_{0}} I(\eta \cup\{z\})}
$$

have the same finite $\mathbb{Z}$-rank. However, the source is free over $\mathbb{Z}$ (because the $\mathbb{Z}$-submodule $J\left(\widehat{\eta} ; M_{0}\right)$ of $\widetilde{E}$ has a set of generators each of which is a linear combination, with coefficients in $\{-1,1\}$, of elements of the obvious (countable) $\mathbb{Z}$-basis of $\widetilde{E}$ ). Hence this surjection is bijective, so $(\mathrm{b})_{n}$ and (c) ${ }_{n}$ follow if $R=\mathbb{Z}$, and then for arbitrary $R$ by base change.

Theorem 2.6. For any $\mathcal{A} \subset \mathbb{P}\left(K^{d+1}\right)$, possibly infinite, the CCSs $\widetilde{A}$ and $A$ satisfy $\mathcal{S}(k)$ for any $1 \leqslant k \leqslant d$.

Proof. The condition $\mathcal{S}(k)$ for $\widetilde{A}$ requires that for any pointed $(k-1)$-simplex $\widehat{\eta}$ and any subset $M_{0} \subset N_{\widehat{\eta}}$ which is stable with respect to $\widehat{\eta}$ the sequence

$$
\widetilde{A}(\eta) \longrightarrow \prod_{z \in M_{0}} \widetilde{A}(\eta \cup\{z\}) \longrightarrow \prod_{\substack{z_{1}, z_{2} \in M_{0} \\\left\{z_{1}, z_{2}\right\} \in X^{1}}} \widetilde{A}\left(\eta \cup\left\{z_{1}, z_{2}\right\}\right)
$$

is exact. For fixed $\widehat{\eta}$ we may pass to a suitable finite subset of $\mathcal{A}$ without changing any of the involved groups. Hence we are in the situation considered above and what we need to show is precisely 


\section{E. Grosse-KLÖNnE}

the exactness of $K\left(\widehat{\eta}, M_{0}\right)$ at its first nontrivial degree. This we did in Proposition 2.5. Hence $\widetilde{A}$ satisfies $\mathcal{S}(k)$. However, then $A$ also satisfies $\mathcal{S}(k)$ because of the split exact sequence (3).

Corollary 2.7. The CCS $\widetilde{A}$ and $A$ on $X$ are acyclic in positive degrees: for any $k \geqslant 1$ we have $H^{k}(X, A)=0$ and $H^{k}(X, \widetilde{A})=0$.

Proof. See Theorems 1.2 and 2.6.

For the rest of this section we assume $\operatorname{char}(K)=0$ and take $\mathcal{A}=\mathbb{P}\left(K^{d+1}\right)$. We write $A_{R}$ instead of $A$ in order to specify the chosen base ring $R$. Let $\Omega_{K}^{(d+1)}$ be Drinfel'd's symmetric space of dimension $d$ over $K$. This is the $K$-rigid space obtained by removing all $K$-rational hyperplanes from projective $d$-space $\mathbb{P}_{K}^{d}$. There is a natural $\mathrm{GL}_{d+1}(K)$-equivariant reduction map

$$
r: \Omega_{K}^{(d+1)} \longrightarrow X
$$

(see, e.g., [deS01] for the precise meaning of $r$ ). For a simplex $\sigma$ of $X$ let $] \sigma\left[=r^{-1}(\operatorname{Star}(\sigma))\right.$, the preimage in $\Omega_{K}^{(d+1)}$ of the star of $\sigma$ : the star of $\sigma$ is the union of the open simplices whose closure contains $\sigma$. This $] \sigma$ [ is an admissible open subset of $\Omega_{K}^{(d+1)}$, and the collection of all the $] \sigma$ [ forms an admissible open covering of $\Omega_{K}^{(d+1)}$.

Proposition 2.8 (de Shalit) [deS01]. For a simplex $\sigma$ of $X$ denote by $H_{d R}^{k}(] \sigma[)$ the $k$ th de Rham cohomology group of the $K$-rigid space $] \sigma[$. There is a natural isomorphism

$$
H_{d R}^{k}(] \sigma[) \cong A_{K}^{k}(\sigma)
$$

Corollary 2.9 .

(1) (Local acyclicity.) Let $\sigma$ be a simplex. For any $k \geqslant 0$ the sequence

$$
0 \longrightarrow H_{d R}^{k}\left(\bigcup_{x \in \sigma}\right] x[) \longrightarrow \prod_{x \in \sigma} H_{d R}^{k}(] x[) \longrightarrow \prod_{\substack{\tau \in X^{1} \\ \tau \subset \sigma}} H_{d R}^{k}(] \tau[) \longrightarrow \cdots
$$

is exact.

(2) (Global acyclicity, de Shalit.) The sequence

$$
0 \longrightarrow H_{d R}^{k}\left(\Omega_{K}^{(d+1)}\right) \longrightarrow \prod_{x \in X^{0}} H_{d R}^{k}(] x[) \longrightarrow \prod_{\sigma \in X^{1}} H_{d R}^{k}(] \sigma[) \longrightarrow \cdots
$$

is exact.

Proof. (1) Choose a vertex $x \in \sigma$. Then $M_{0}=\sigma-\{x\}$ is (as a set of vertices) stable with respect to $x$. Since the CCS $A_{K}$ satisfies $\mathcal{S}(k)$ for any $k$, we derive just as in the proof of Proposition 2.5 that the sequence

$$
\prod_{z \in \sigma} A_{K}(z) \longrightarrow \prod_{\substack{\tau \in X^{1} \\ \tau \subset \sigma}} A_{K}(\tau) \longrightarrow \cdots
$$

is exact. Inserting Proposition 2.8 it becomes the exact sequence

$$
\prod_{x \in \sigma} H_{d R}^{k}(] x[) \longrightarrow \prod_{\substack{\tau \in X^{1} \\ \tau \subset \sigma}} H_{d R}^{k}(] \tau[) \longrightarrow \cdots
$$

On the other hand, we have the spectral sequence

$$
E_{1}^{r s}=\prod_{\substack{\tau \in X^{r} \\ \tau \subset \sigma}} H_{d R}^{s}(] \tau[) \Longrightarrow H_{d R}^{s+r}\left(\bigcup_{x \in \sigma}\right] x[)
$$


Together (1) follows. The proof of (2) works the same way, using Corollary 2.7 instead of Theorem 2.6.

Corollary 2.9 gives a precise expression of $H_{d R}^{k}\left(\Omega_{K}^{(d+1)}\right)$ through all the $H_{d R}^{k}(] \sigma[)$. The natural $\mathbb{Z}$-structures $A_{\mathbb{Z}}^{k}(\sigma)$ in the $A_{K}^{k}(\sigma)$ provide natural $\mathbb{Z}$-structures $H_{\mathbb{Z}}^{k}(] \sigma[)$ in the $H_{d R}^{k}(] \sigma[)$; hence the $\mathrm{GL}_{d+1}(K)$-stable subgroup

$$
H_{\mathbb{Z}}^{k}\left(\Omega_{K}^{(d+1)}\right)=\operatorname{Ker}\left[\prod_{x \in X^{0}} H_{\mathbb{Z}}^{k}(] x[) \longrightarrow \prod_{\sigma \in X^{1}} H_{\mathbb{Z}}^{k}(] \sigma[)\right]
$$

of $H_{d R}^{k}\left(\Omega_{K}^{(d+1)}\right)$. Now our Corollary 2.7 expresses $H_{\mathbb{Z}}^{k}\left(\Omega_{K}^{(d+1)}\right)$ precisely through the local terms $A_{\mathbb{Z}}^{k}(\sigma)$ : it tells us that $H_{\mathbb{Z}}^{k}\left(\Omega_{K}^{(d+1)}\right)$ is quasiisomorphic with the complex

$$
\prod_{x \in X^{0}} H_{\mathbb{Z}}^{k}(] x[) \longrightarrow \prod_{\sigma \in X^{1}} H_{\mathbb{Z}}^{k}(] \sigma[) \longrightarrow \prod_{\sigma \in X^{2}} H_{\mathbb{Z}}^{k}(] \sigma[) \longrightarrow \cdots
$$

Let us explain why this should have an application to a challenging problem on $p$-adic AbelJacobi mappings raised by Raskind and Xarles [RX03]. Let $\Gamma \subset \mathrm{PGL}_{d+1}(K)$ be a cocompact discrete subgroup such that the quotient $Y=\Gamma \backslash \Omega_{K}^{(d+1)}$, a smooth projective $K$-scheme, has strictly semistable reduction. For $1 \leqslant k \leqslant d$, Raskind and Xarles associate to $Y$ a certain rigid analytic torus $J^{k}(Y)$, a ' $p$-adic intermediate Jacobian' $\left(J^{1}(Y)\right.$ is the Picard variety of $Y$ and $J^{d}(Y)$ is the Albanese variety of $Y)$. The device for the construction of $J^{k}(Y)$ is a canonical $\mathbb{Z}$-structure in the graded pieces $\mathrm{Gr}_{*}^{M} H^{k}(Y)$ of the monodromy filtration on the cohomology $H^{k}(Y)$ of $Y$ - both $\ell$-adic $(\ell \neq p)$ and $\log$ crystalline $(\cong$ de Rham) cohomology. This $\mathbb{Z}$-structure results from the fact that for any component intersection $Z$ of the reduction of $Y$ (so each $Z$ is a smooth projective $k$-scheme), the cycle map

$$
C H^{k}\left(Z \times_{k} \bar{k}\right) \otimes W(-k) / \text { tors } \longrightarrow H_{\text {crys }}^{2 k}\left(Z \times_{k} \bar{k} / W\right) / \text { tors }
$$

is bijective (here $W(-k)$ is the ring of Witt vectors $W$ with the action of Frobenius multiplied by $p^{k}$ ); similarly for $\ell$-adic cohomology, as was recently proved by Ito [Ito03]. That is, the $\mathbb{Z}$-structure is essentially given by the collection of Chow groups for all $Z$. Then they define an Abel-Jacobi mapping

$$
C H^{k}(Y)_{\text {hom }} \longrightarrow J^{k}(Y)(K)
$$

with $C H^{k}(Y)_{\text {hom }}$ the group of cycles that are homologically equivalent to zero, using the $\ell$-adic $\left(\ell \neq p\right.$ and $\ell=p$ ) Abel-Jabobi mapping which involves the Galois cohomology groups $H_{g}^{1}(K, \cdot)$ defined by Bloch and Kato. As they point out, it would be helpful to define the Abel-Jabobi mapping by analytic means.

We expect that such a definition involves $p$-adic integration of cycles on the (contractible!) $K$-rigid space $\Omega_{K}^{(d+1)}$, similar to Besser's $p$-adic integration on $K$-varieties with good reduction. The link would be the covering spectral sequence

$$
E_{2}^{r s}=H^{r}\left(\Gamma, H^{s}\left(\Omega_{K}^{(d+1)}\right)\right) \Longrightarrow H^{r+s}(Y)
$$

(which exists for both $\ell$-adic $(\ell \neq p)$ and de Rham cohomology). Indeed, we know that the associated filtration on $H^{k}(Y)$ is the monodromy filtration [deS05, Gro, Ito03]), therefore the $\mathbb{Z}$-structure $H_{\mathbb{Z}}^{k}\left(\Omega_{K}^{(d+1)}\right)$ in $H^{k}\left(\Omega_{K}^{(d+1)}\right)$ gives a $\mathbb{Z}$-structure in $\mathrm{Gr}_{*}^{M} H^{k}(Y)$. A comparison with that of Raskind and Xarles probably needs the resolution (10): the component intersections $Z$ considered by them correspond precisely to the simplices of the quotient simplicial complex $\Gamma \backslash X$. 


\section{E. Grosse-KLÖNnE}

\section{Local systems arising from representations}

Let $K, \mathcal{O}_{K}, \pi, k, X$ and its orientation be as in $\S 2$. We fix a natural number $n \geqslant 1$ and let

$$
U=U^{(n)}=\left\{g \in \mathrm{GL}_{d+1}\left(\mathcal{O}_{K}\right) \mid g \equiv 1 \bmod \pi^{n}\right\}
$$

denote the principal congruence subgroup of level $n$ in $G$. For a vertex $x \in X^{0}$ we let

$$
U_{x}=U_{x}^{(n)}=g U g^{-1} \quad \text { if } x=g\left(\left[\mathcal{O}_{K}^{d+1}\right]\right) \text { for some } g \in G
$$

and for a simplex $\tau=\left\{x_{1}, \ldots, x_{k}\right\}$ we let

$$
U_{\tau}=\text { the subgroup generated by } U_{x_{1}} \cup \cdots \cup U_{x_{k}} \text {. }
$$

This is a pro- $p$-group, $p=\operatorname{char}(k)$.

Lemma 3.1. Suppose the lattices $L_{z}, L_{x_{1}}$ and $L_{x_{2}}$ represent vertices $z, x_{1}$ and $x_{2}$ in $X^{0}$ such that both $x_{1}$ and $x_{2}$ are incident to $z$ and such that $L_{z}=L_{x_{1}} \cap L_{x_{2}}$. Then $U_{z} \subset U_{x_{1}} U_{x_{2}}$.

Proof. Applying a suitable $g \in G$ we may assume that $L_{z}=\mathcal{O}_{K}^{d+1}$ and $L_{x_{s}}=t^{s} \mathcal{O}_{K}^{d+1}$ for diagonal matrices id $\neq t^{s}=\left(t_{0}^{s}, \ldots, t_{d}^{s}\right)$ satisfying $\{1\} \subset\left\{t_{j}^{1}, t_{j}^{2}\right\} \subset\left\{1, \pi^{-1}\right\}$ for all $0 \leqslant j \leqslant d$. However, then $U_{z}=U$ as defined above, and $U_{x_{s}}=t^{s} U\left(t^{s}\right)^{-1}$ and an easy matrix argument gives the claim.

Proposition I.3.1 in [SS97] significantly strengthens Lemma 3.1. It is this interpolation property of the groups $U_{x}$ which also underlies the acyclicity proof in [SS93] and the much more general theory in [SS97].

Let $V$ be a smooth representation of $G$ on a (not necessarily free) $\mathbb{Z}[1 / p]$-module $V$ which is generated, as a $G$-representation, by its $U$-fixed vectors. Because of $U_{\sigma} \subset U_{\tau}$ if $\sigma \subset \tau$ we can form the HCS $\underline{V}=\left(V^{U_{\tau}}\right)$ of subspaces of fixed vectors

$$
V^{U_{\tau}}=\left\{v \in V \mid g v=g \text { for all } g \in U_{\tau}\right\}
$$

with the obvious inclusions as transition maps. In the special case where our $V$ is a $G$ representation on a $\mathbb{C}$-vector space (not just on a $\mathbb{Z}[1 / p]$-module), the following theorem (and its version for $n=1$ ) was proved in [SS93].

Theorem 3.2. Suppose $n>1$. Then the chain complex $C \cdot(X, \underline{V})$ is a resolution of $V$.

Proof. To see $H_{k}(X, \underline{V})=0$ for $k \geqslant 1$ it suffices, by Theorem 1.7, to prove $\mathcal{S}^{*}(k)$, i.e. to prove that for any pointed $(k-1)$-simplex $\widehat{\eta}$ with underlying $(k-1)$-simplex $\eta$ and for any subset $M_{0}$ of $N_{\widehat{\eta}}$ which is stable with respect to $\widehat{\eta}$, the sequence

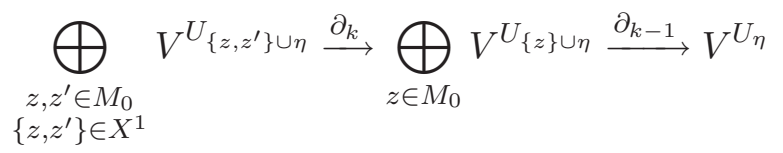

is exact. We use induction on $\left|M_{0}\right|$. If $M_{0}$ is non-empty choose a $y \in M_{0}$ for which $L_{y}$ is maximal, i.e. there is no $z \in M_{0}$ with $L_{y} \subsetneq L_{z}$. Then $M_{1}=M_{0}-\{y\}$ is stable with respect to $\widehat{\eta}$. Letting

$$
M_{1}^{\prime}=\left\{z^{\prime} \in M_{1} \mid\left\{y, z^{\prime}\right\} \in X^{1}\right\}
$$

we first claim that

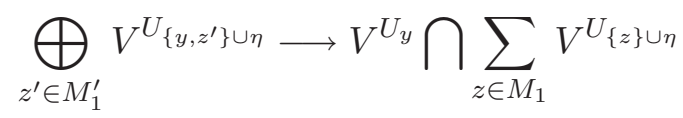

is surjective. Let $v=\sum_{z \in M_{1}} v_{z}$ be an element of the right-hand side with $v_{z} \in V^{U_{\{z\} \cup \eta}}$ for all $z \in M_{1}$. Since $V$ is smooth we can find a (finitely generated) submodule $V^{\prime}$ of $V$ containing $v$ which 


\section{ACYCLIC COEFFICIENT SYSTEMS ON BUILDINGS}

is stable under $U_{\eta}$ and $U_{z}$ for all $z \in M_{0}$. The action of $U_{y}$ on $V^{\prime}$ factors through a finite quotient $\bar{U}_{y}$ of $U_{y}$. Since $v$ is fixed by $\bar{U}_{y}$ it follows that

$$
v=\frac{1}{\left|\bar{U}_{y}\right|} \sum_{g \in \bar{U}_{y}} g \cdot v=\sum_{z \in M_{1}} \frac{1}{\left|\bar{U}_{y}\right|} \sum_{g \in \bar{U}_{y}} g \cdot v_{z} .
$$

Since $M_{0}$ is stable, there exists for any $z \in M_{1}$ a $z^{\prime} \in M_{1}^{\prime}$ such that $L_{z^{\prime}}=L_{z} \cap L_{y}$. It will be enough

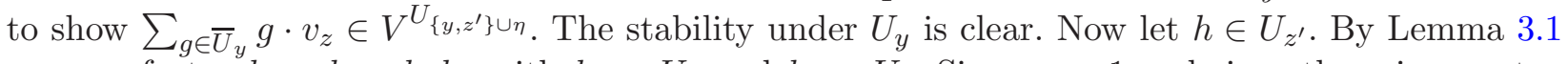
we may factor $h$ as $h=h_{y} h_{z}$ with $h_{y} \in U_{y}$ and $h_{z} \in U_{z}$. Since $n>1$ and since there is a vertex incident to both $z$ and $y$ we have $g^{-1} U_{z} g=U_{z}$ for any $g \in U_{y}$, hence $h_{z} g=g h_{z}^{g}$ with $h_{z}^{g} \in U_{z}$. Thus

$$
h \sum_{g \in \bar{U}_{y}} g \cdot v_{z}=h_{y} \sum_{g \in \bar{U}_{y}} g h_{z}^{g} \cdot v_{z}=\sum_{g \in \bar{U}_{y}} g \cdot v_{z},
$$

i.e. $\sum_{g \in \bar{U}_{y}} g \cdot v_{z}$ is stable under $U_{z^{\prime}}$. Finally let $h \in U_{x}$ for some $x \in \eta$. Since $x$ and $y$ are incident we have $g^{-1} U_{x} g=U_{x}$, hence there is for any $g \in U_{y}$ a $h^{g} \in U_{x}$ with $h g=g h^{g}$. Then

$$
h \sum_{g \in \bar{U}_{y}} g \cdot v_{z}=\sum_{g \in \bar{U}_{y}} g h^{g} \cdot v_{z}=\sum_{g \in \bar{U}_{y}} g \cdot v_{z}
$$

so we have shown stability under $U_{\eta}$. The surjectivity of (11) is proven. Now let $c=\left(c_{z}\right)_{z \in M_{0}}$ be an element of $\operatorname{Ker}\left(\partial_{k-1}\right)$. Then necessarily

$$
c_{y} \in V^{U_{y}} \cap \sum_{z \in M_{1}} V^{U_{\{z\} \cup \eta}}
$$

By the surjectivity of (11) we may therefore modify $c$ by an element of $\operatorname{Im}\left(\partial_{k}\right)$ such that for the new $c=\left(c_{z}\right)_{z \in M_{0}} \in \operatorname{Ker}\left(\partial_{k-1}\right)$ we have $c_{y}=0$. However, then the induction hypothesis, applied to $M_{1}$, tells us that after another such modification we can achieve $c=0$. We have shown that $C .(X, \underline{V})$ is exact in positive degrees. It remains to observe that the hypothesis that $V$ is generated by $V^{U}$ is equivalent with the surjectivity of

$$
C_{0}(X, \underline{V})=\bigoplus_{x \in X^{0}} V^{U_{x}} \longrightarrow V .
$$

\section{ACKNowledGements}

I am very grateful to Peter Schneider whose comments helped me to eliminate inaccuracies and to streamline presentation, terminology and notations. I thank Ehud de Shalit and Annette Werner for related discussions. Thanks also go to the referee for his careful reading and for supplying a simple proof for Lemma 2.3 .

\section{REFERENCES}

Alo G. Alon, Cohomology of local systems coming from p-adic hyperplane arrangements, Israel J. Math., to appear.

Bro89 K. S. Brown, Buildings (Springer, New York, 1989).

deS01 E. de Shalit, Residues on buildings and de Rham cohomology of p-adic symmetric domains, Duke Math. J. 106 (2001), 123-191.

deS05 E. de Shalit, The p-adic monodromy-weight conjecture for p-adically uniformized varieties, Compositio Math. 141 (2005), 101-120.

Gro E. Grosse-Klönne, Frobenius and monodromy operators in rigid analysis, and Drinfel'd's symmetric space, J. Algebraic Geom., to appear. 


\section{ACYCLIC COEFFICIENT SYSTEMS ON BUILDINGS}

Gro04 E. Grosse-Klönne, Integral structures in the p-adic holomorphic discrete series, Preprint (2004).

Ito03 T. Ito, Weight-monodromy conjecture for p-adically uniformized varieties, Preprint (2003).

RX03 W. Raskind and X. Xarles, On the étale cohomology of algebraic varieties with totally degenerate reduction over p-adic fields, Preprint (2003).

Sch92 P. Schneider, The cohomology of local systems on p-adically uniformized varieties, Math. Ann. 293 (1992), 623-650.

SS93 P. Schneider and U. Stuhler, Resolutions for smooth representations of the general linear group over a local field, J. reine angew. Math. 436 (1993), 19-32.

SS97 P. Schneider and U. Stuhler, Representation theory and sheaves on the Bruhat-Tits building, Publ. Math. Inst. Hautes Études Sci. 85 (1997), 97-191.

Elmar Grosse-Klönne klonne@math.uni-muenster.de

Mathematisches Institut der Universität Münster, Einsteinstrasse 62, D-48149 Münster, Germany 